\title{
Anti-inflammatory effects of tetradecylthioacetic acid (TTA) in macrophage-like cells from Atlantic salmon (Salmo salar L.)
}

Fabian Grammes ${ }^{1,2}$ and Harald Takle $2,3^{*}$

\begin{abstract}
Background: Commercial Atlantic salmon is fed diets with high fat levels to promote fast and cost-effective growth. To avoid negative impact of obesity, food additives that stimulate fat metabolism and immune function are of high interest. TTA, tetradecylthioacetic acid, is a synthetic fatty acid that stimulates mitochondrial $\beta$-oxidation most likely by activation of peroxysome proliferator-activated receptors (PPARs). PPARs are important transcription factors regulating multiple functions including fat metabolism and immune responses. Atlantic salmon experiments have shown that TTA supplemented diets significantly reduce mortality during natural outbreaks of viral diseases, suggesting a modulatory role of the immune system.
\end{abstract}

Results: To gain new insights into TTA effects on the Atlantic salmon immune system, a factorial, high-throughput microarray experiment was conducted using a 44K oligo nucleotide salmon microarray SIQ2.0 and the Atlantic salmon macrophage-like cell line ASK. The experiment was used to determine the transcriptional effects of TTA, the effects of TTA in poly(l:C) elicited cells and the effects of pretreating the cells with TTA. The expression patterns revealed that a large proportion of genes regulated by TTA were related to lipid metabolism and increased mitochondrial $\beta$-oxidation. In addition we found that for a subset of genes TTA antagonized the transcriptional effects of poly $(I: C)$. This, together with the results from qRT-PCR showing an increased transcription of antiinflammatory IL10 by TTA, indicates anti-inflammatory effects.

Conclusions: We demonstrate that TTA has significant effects on macrophage-like salmon cells that are challenged by the artificial dsRNA poly $(I: C)$. The immune stimulatory effect of TTA in macrophages involves increased lipid metabolism and suppressed inflammatory status. Thus, suggesting that TA directs the macrophage-like cells towards alternative, anti-inflammatory, activation. This has positive implications for TTA as a feed additive.

\section{Background}

In commercial Atlantic salmon (Salmo salar L.) aquaculture, diets usually contain high levels of fat as it provides an inexpensive source of energy and promotes rapid growth. However, high levels of dietary fat cause excess fat to be deposited in the tissues, most notably in muscle, liver and as visceral fat [1]. Thereby the diet may affect the general health of the Atlantic salmon and also the quality of the filet as the final product [2]. In order to reduce the negative side effects of high fat diets, several studies have experimented with food additives promoting fat catabolism. Tetradecylthioacetic acid

\footnotetext{
* Correspondence: harald.takle@nofima.no

${ }^{2}$ NOFIMA, P.O. Box 5010, N-1432 s, Norway

Full list of author information is available at the end of the article
}

(TTA) is one of these additives, considered to be highly interesting.

TTA is a synthetic fatty acid with a sulfur substitution at the 3rd position in the carbon chain. The chemical properties of TTA resemble those of a normal fatty acid of similar length. What makes TTA interesting is that the sulfur atom makes it resistant towards further catabolism via $\beta$-oxidation and that TTA serves as activator for all peroxysome proliferator-activated receptor (PPAR) subtypes [3,4]. PPARs are important transcription factors regulating multiple functions, most notably fat metabolism and immune responses (reviewed in [5]). TTA has been reported to induce pleiotropic effects in mammals. These effects include increased mitochondrial $\beta$-oxidation in muscle and liver, decreased plasma lipid

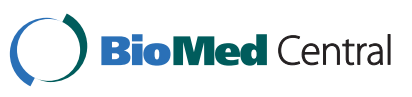


levels as well as antioxidant and anti-inflammatory effects (reviewed in [6,7]). It appears that most of the metabolic effects of TTA are mediated through activation of PPARs.

Studies addressing metabolic effects of TTA in fish are scarce. Nevertheless, the studies that have been conducted with members of the salmonidae family indicate similar metabolic effects of TTA in fish by showing increased lipid metabolism in liver $[8,9]$ and muscle [10]. In contrast to mammals, high levels of dietary TTA have been reported to induce mortality in Atlantic salmon $[9,11]$ but not in Rainbow trout (Oncorhynchus mykiss Walbaum) [8]. It has been hypothesized that the mortality may be related to accumulation of TTA-metabolites in the kidney at low temperatures [12].

To date only one study investigated immune modulatory functions of TTA in Atlantic salmon, showing that TTA reduces the production of leukotriene $\mathrm{B} 4\left(\mathrm{LTB}_{4}\right)$ and prostaglandin $\mathrm{E}_{2}\left(\mathrm{PGE}_{2}\right)$ in headkidney macrophages isolated from TTA fed fish [12]. It should be noted that the reduction was found only to be significant for cells cultured at $5^{\circ} \mathrm{C}$, but not in those maintained at $12^{\circ} \mathrm{C}$ [12]. Intriguingly, two studies, both reporting significant reduced mortality in Atlantic salmon pre fed with a low-level TTA supplemented diet during natural outbreaks of viral diseases in sea $[10,13]$, indicate important immune modulatory effects of TTA. Hence, TTA may be of interest as a feed additive in Atlantic salmon aquaculture.

To address immune modulatory functions of TTA in Atlantic salmon we conducted a "genome wide" microarray approach in a factorial designed experiment to identify transcriptional effects of TTA using the established, macrophage-like cell line ASK [14]. The cell line was chosen because macrophages pose a key role in the innate immunity, which is considered to be of primary importance to fight off pathogens since it can take weeks to mount an acquired immune response in poikilothermic fish [15]. Macrophages posses a remarkable plasticity that allows them to change their phenotype according to environmental signals. In vitro two major macrophage phenotypes have been characterized. Classically activated macrophages displaying a pro-inflammatory profile and alternative activated macrophages showing anti-inflammatory properties [16]. Macrophages recognize invading pathogens via pattern recognition receptors that bind conserved pathogen-associated molecular patterns (PAMPs). Activation of macrophages via PAMPs induces the classical activated phenotype. Because most viral pathogens produce dsRNA during one part of their replication [17], we decided to use the double stranded RNA analogue poly(I:C), a frequently used PAMP to simulate infection with a viral pathogen in our experiment.
A large proportion of the genes regulated by TTA was related to increased lipid metabolism and were also activated by TTA in poly(I:C) elicited ASK cells. The main effect seen for TTA in poly(I:C) elicited cells was to antagonize the induction of a subset of poly(I:C) dependent genes. Further we found increased expression of anti-inflammatory IL10 and Krueppel-like factor 11 (KLF11). Thus the results indicate that TTA polarizes the macrophage-like cells towards the alternative activated state.

\section{Results}

We used the permanent Atlantic salmon macrophagelike cell line ASK in a factorial designed experiment to study transcriptional effects of TTA. An overview of the experimental design is given in Table 1. RNA from two biological replicates was used in the microarray analysis with the Atlantic salmon SIQ2.0 microarray [18] in a one-color setup, resulting in totally 16 arrays. One array did not pass the quality control analysis and was subsequently excluded from the final data set (Table 1, indicated $\left.b^{*}\right)$. To identify differentially expressed (DE) probes, a linear model (Equation 2), containing the main effects was fitted to each probe set. Subsequently we extracted specific contrasts (Table 2), using the Bioconductor package "limma" [19].

Pretreatment with TTA (preTTA vs. CTR) significantly altered the expression of 121 probes (108 genes), while treatment with TTA (TTA vs. CTR) affected 49 probes (41 genes) (Table 3). Stimulating the cells with poly(I:C) showed the strongest effect with regard to number of DE probes and $\log _{2} \mathrm{FC}$ with $3636 \mathrm{DE}$ probes $(2785$ genes) in the contrast IC vs. CTR. We observed significant alteration of 3487 probes (2697 genes) when treating the cells with TTA and poly(I:C) simultaneously (TTAIC vs. CTR). 92 probes (70 genes) were DE when comparing the concordant TTA and poly(I:C) treatment vs. poly(I:C) treatment (IC vs. TTAIC), indicating that

\section{Table 1 Experimental design}

\begin{tabular}{|c|c|c|c|}
\hline Pretreatment & Treatment & biol. repl. ${ }^{a}$ & array $^{b}$ \\
\hline \multirow[t]{4}{*}{ Control } & Control & 4 & 2 \\
\hline & TTA & 4 & 2 \\
\hline & TTA+poly(l:C) & 4 & 2 \\
\hline & poly $(1: C)$ & 4 & 2 \\
\hline \multirow[t]{4}{*}{ TTA } & Control & 4 & 2 \\
\hline & TTA & 4 & 2 \\
\hline & TTA+poly $(I: C)$ & 4 & 2 \\
\hline & poly $(\mathrm{l}: \mathrm{C})$ & 4 & $2^{*}$ \\
\hline
\end{tabular}

a Replicates, using different passages of the cells

${ }^{b}$ Number of replicates used in the array experiment

* One of the arrays did not pass the quality control and was excluded from further analysis 
Table 2 Specific contrasts

\begin{tabular}{|c|c|c|}
\hline Contrast & $H_{0}$ to test & Interpretation when $H_{0}$ is rejected and the corresponding $\log _{2} \mathrm{FC}$ passes the cut-off \\
\hline preTtA vs. CTR & $H_{0}: P_{T T A}=P_{\text {contr }}$ & DE in: Samples pretreated with TTA vs. pretreatment Control \\
\hline TTA vs. CTR & $H_{0}: T_{T A A}=T_{\text {contr }}$ & DE in: Samples treated with TTA vs. Control treated \\
\hline TTAIC vs. CTR & $H_{0}: T_{T T A+p o l y: C}=T_{\text {contr }}$ & DE in: Samples treated with TTA+poly(l:C) vs. Control treated \\
\hline IC vs. CTR & $H_{0}: T_{\text {poly: }: C}=T_{\text {contr }}$ & DE in: Samples treated with poly(l:C) vs. Control treated \\
\hline IC vs. TTAIC & $H_{0}: T_{\text {TTA+poly: }: C}=T_{\text {poly: }: C}$ & DE in: Samples treated with TTA+poly $(I: C)$ vs. poly(l:C) treated \\
\hline
\end{tabular}

Contrasts, extracted for each probe after the full linear model (Equation 2) was fitted to the data.

TTA exerts effects on transcription in poly(I:C) challenged cells.

In this study we were primarily interested in transcriptional effects related to TTA. Thus, we will focus for the rest of the paper on the three TTA related contrasts: preTTA vs. CTR, TTA vs. CTR and IC vs. TTAIC. However, a comprehensive list of all DE probes for each contrast can be found in Additional file 1, Table S1.

The number of DE probes and their overlap between the different contrasts is shown in Figure 1.

\section{Transcriptional effects in IC vs. TTAIC}

92 probes were DE when comparing ASK cells treated with poly(I:C) alone vs. cells that received concordant TTA and poly(I:C) treatment (Figure 1 and 2). Considering that $\operatorname{poly}(\mathrm{I}: \mathrm{C})$ treatment alone significantly altered the expression of 3636 probes (Figure 1), the effects of TTA under poly(I:C) elicitation may be considered relatively small.

We observed that after hierarchical clustering of the DE probes for the IC vs. TTAIC contrast the genes could be separated into three major clusters (Figure 2). First, probes showing mostly a moderate down-regulation in response to poly $(\mathrm{I}: \mathrm{C})$, but a generally stronger down-regulation upon concordant TTA poly(I:C) treatment (cluster.1). Second, probes that were up-regulated by TTA, but down-regulated by poly(I:C) treatment

Table 3 Summary of significant probes identified by the different contrasts

\begin{tabular}{cllll}
\hline Contrast & UP/DOWN & Probes & $\begin{array}{l}\text { Probes } \\
\text { (Ifc >1) }\end{array}$ & $\begin{array}{l}\text { Probes } \\
\text { (Ifc >2) }\end{array}$ \\
\hline Pretreatment & & & & \\
preTTA vs. CTR & UP & 119 & 4 & 0 \\
& DOWN & 2 & 0 & 0 \\
\hline Treatment & & & & \\
TTA vs. CTR & UP & 27 & 6 & 0 \\
& DOWN & 22 & 1 & 0 \\
TTAIC vs. CTR & UP & 1043 & 476 & 250 \\
& DOWN & 2444 & 764 & 72 \\
IC vs. CTR & UP & 1491 & 594 & 227 \\
& DOWN & 2145 & 787 & 80 \\
IC vs. TTAIC & UP & 22 & 4 & 0 \\
& DOWN & 70 & 9 & 0 \\
\hline
\end{tabular}

(cluster.2). And third, probes that were less up-regulated when treated with TTA in combination with poly(I:C) (cluster.3). Especially for the second and the third cluster of probes we observed profound antagonistic effects for TTA in poly(I:C) elicited macrophages.

We found that cluster. 2 contained mostly genes that were related to fat metabolism, and that most of them were shared with the TTA vs CTR contrast (see next section). Genes of particular interest were: ANGPTL4, as the highest ranking gene, Acadm, AGPAT4, THIM, HADHA, PLIN2 and KLF11. Angiopoetin-related protein 4 (ANGPTL4) has been reported, beside having a role in regulating angiogenesis, to act as a important stimulator of lipid metabolism [20]. Acetyl-CoA acyltransferase THIM and the mitochondrial trifunctional enzyme HADHA are enzymes of the mitochondrial $\beta$-oxidation. The gene AGPAT4 encodes an acyltransferase, participating in de-novo phospholipid synthesis. The gene remains yet to be fully characterized, however, other isoforms have been reported to be regulated by PPAR- $\alpha$ [21]. The protein Perilipin 2 (PLIN2) is a lipid droplet associated protein, recently suggested to stimulate

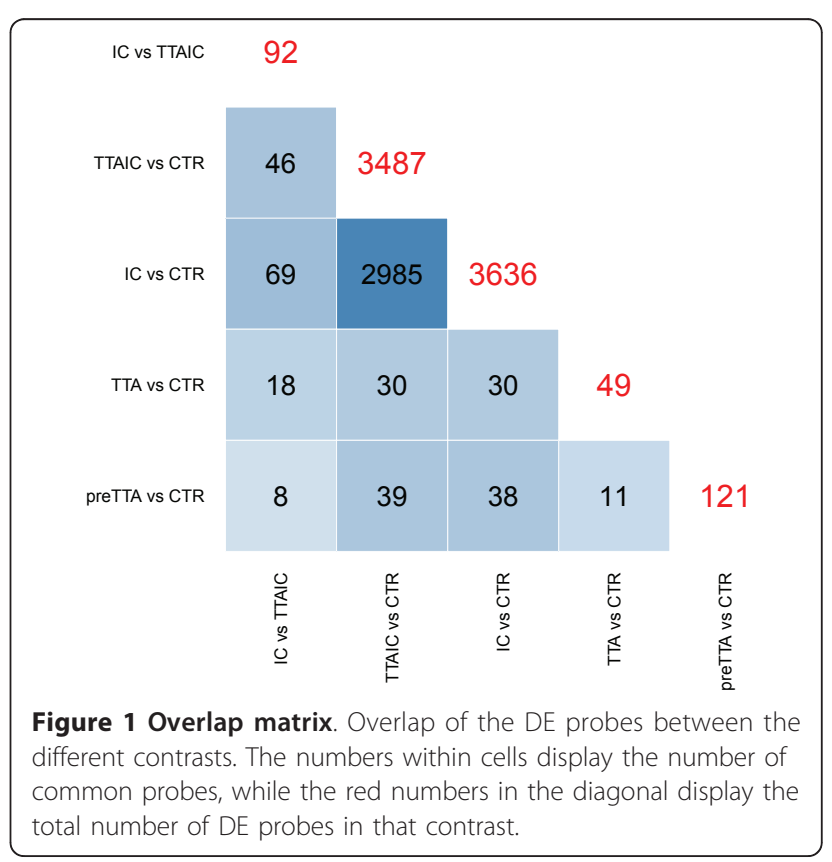




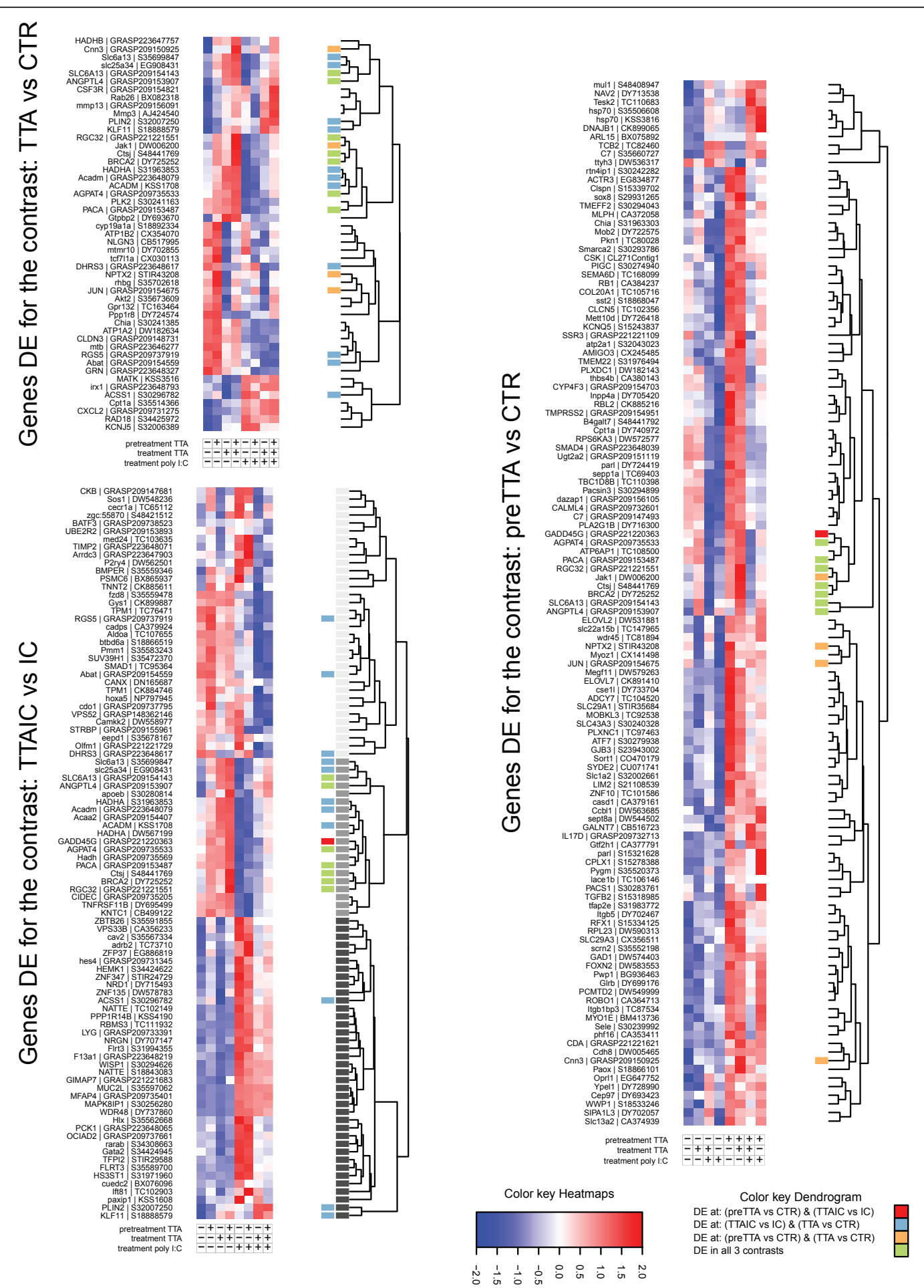

Figure 2 Heatmaps. Heatmaps displaying the relative expression of the probes significant in the three TTA related contrasts (IC vs. TTAIC, TTA vs. CTR and preTTA vs. CTR. The probes were clustered hierarchical using euclidean distance as indicated by the dendrogram on the side. The color code attached to the dendrogram indicates overlap for the corresponding gene between the 3 contrasts. In the dendrogram of the heatmap for contrast IC vs. TTAIC, the three major clusters are highlighted additionally (lightgrey - cluster.1; grey - cluster.2; darkgrey - cluster.3). 
lipolysis in macrophages [22]. Moreover, KLF11 an inhibitor of cellular $\mathrm{PGE}_{2}$ synthesis [23] and APOEB, the teleost homolog to apoE, was found to be strongly upregulated. $\mathrm{PGE}_{2}$ is an important mediator of inflammation and has been previously reported to be down-regulated in response to TTA treatment $[12,24]$. ApoE secreted by macrophages participates in the regulation of cholesterol efflux, reducing the cholesterol-ester accumulation in macrophages attached to the arterial wall [25]. In addition, ApoE in macrophages has been reported to act as suppressor of pro-inflammatory signals and vice versa [26], and is reported to respond to PPAR- $\gamma$ agonists in macrophages [27]. Hence, these results suggest that TTA induces lipid metabolism and also affects lipid mediators of inflammation in poly(I:C) elicited macrophages.

The genes in cluster. 1 and cluster.3, down-regulated for the IC vs. TTAIC contrast, contained several genes with reported functions in immune modulation. The genes encoding Nattectin (NATTE) and Lysozyme G (LYG) both showed a strong increase in transcription in response to poly(I:C), which was attenuated when TTA was added. Nattectin has been identified as C-type lectin in fish [28]. C-type lectins function as pattern recognition receptors, recognizing pathogens and may subsequently opsonize them for phagocytosis. C-type lectins are induced following infection with bacterial [29] and viral [30] pathogens in fish, hence, suggesting host defense properties. Lysozyme is primarily associated with the defense against bacterial pathogens through hydrolyzing $\beta 1,4$ glycoside bounds of peptidoglucans in the bacterial cell wall [31]. Expression of the antiinflammatory marker Pituitary adenylate cyclase-activating polypeptide (PACA) [32], was strongly decreased in the IC vs. CTR contrast, but showed increased expression in the IC vs. TTAIC contrast. A similar pattern was observed for Os-teoprotegerin (TNFRSF11B). Osteoprotegerin is a member of the tumor necrosis receptor superfamily. Initially Osteoprotegrin was characterized for its ability to suppress osteoclast formation [33]. However Osteoprotegrin has also been reported to have a role in controlling inflammation. Exposure of mammalian endothelial cells to inflammatory cytokines has been reported to increase Osteoprotegrin expression, which in turn promotes leucocyte adhesion in vitro and in vivo [34].

\section{Transcriptional effects in TTA vs. CTR}

49 probes were DE in response to treatment with TTA (Figure 1, Figure 2). From these 49 probes we found 27 to be up- and 22 probes to be down-regulated. The transcriptional changes in general were modest, with 6 probes showing an absolute $\log _{2} \mathrm{FC} \geq 1$ (Table 3 ). As shown in Figure 1 (and indicated in the dendrogram of
Figure 2) the overlap to the previously described IC vs. TTAIC contrast was $37 \%$, demonstrating that TTA affects a slightly different subsets of genes when applied alone to ASK cells.

Treatment with TTA generally increased the expression of genes involved in lipid metabolism. The gene showing the highest $\log _{2} \mathrm{FC}$ in this contrast was Angiopoetin-related protein 4 (ANGPTL4). Further we found a couple of genes encoding crucial enzymes for mitochondrial $\beta$-oxidation. We found carnitine palmitoyltransferase $1 A(C p t 1 a)$, the acyl-CoA dehydrogenase (ACADM ) and mitochondrial trifunctional enzyme $(H A D H B)$ to be up-regulated. Also we observed increased expression of the gene encoding the lipid particle associated protein PLIN2 and short-chain dehydrogenase/reductase DHRS3. Thus, indicating increased lipid metabolism in ASK cells following TTA treatment.

We observed TTA regulation of a number of genes associated to anti-inflammatory effects. The second highest ranking gene up-regulated was the $\mathrm{PGE}_{2}$ inhibitor KLF11 [23]. Moreover we observed attenuated transcription of Grannulins (GRN)/progrannulin and the neuropeptide pituitary adenylate cyclase-activating polypeptide (PACA). Both genes are reported to exhibit antiinflammatory effects. Macrophages isolated from mice deficient for the Grannulin gene showed higher transcription levels of pro-inflammatory cytokines [35], indicating an anti-inflammatory role for progrannulin. PACA has been identified to exert anti-inflammatory effects [32]. Increased expression of the matrix metalloproteinases $M M P 3, M M P 13$ and chemokine ligand 2 (CXCL2) was found in TTA treated ASK cells. Matrix metalloproteinases (MMPs) possess pleiotropic functions such as acting as matrix degrading enzymes, immunomodulary functions and have been suggested to be required for the migration of inflammatory cells [36]. CXCL2 has been implicated to act as chemotactic or activating factor for monocytes [37] and was further found to be highly induced by poly(I:C) treatment. Thus suggesting an increased potential for cell migration induced by TTA.

Further, megakaryocyte-associated tyrosine kinase (MATK ) and the transcription factor AP-1 (JUN ) proofed to be down-regulated. MATK is a nonreceptor tyrosine kinase that negatively regulates the activity of the Src family protein kinases - key regulators in signal transduction. A study showed higher levels of interferon $-\gamma$ in MATK K.O mice challenged with antigens, thus suggesting a function for MATK in the immunological response [38]. JUN is an important transcription factor responding to cytokines and stress and has been reported to be up-regulated in response to viral challenges in Atlantic salmon [39,40]. Further we found a decreased expression of NPTX2, a member of the 
pentraxin superfamily that are known to act as pattern recognition receptors and can facilitate pathogen recognition and removal [15].

\section{Transcriptional effects in preTTA vs. CTR}

In the two experiments showing lower mortality in Atlantic salmon fed with TTA upon natural (viral) disease outbreak, the TTA feeding period ended before the onset of disease related mortality $[10,13]$. So we speculated that TTA might have long-term effects on the immunity in Atlantic salmon. To identify these effects we included the factor pretreatment in the experimental design. We found that pretreatment with TTA caused DE of 121 probes, which with the exception of two probes, all showed increased transcription (Table 3). The list of DE probes showed relatively little overlap with the two previously described TTA contrasts (7\% and $9 \%$ to the contrasts IC vs. TTAIC and TTA vs. CTR respectively, Figure 2). Hence, suggesting that TTA pretreatment affects a different subset of genes. However, 38 of the probes in this contrast were also DE in the contrast IC vs. CTR (Figure 1). It should be noted that, similar to the effects observed in IC vs. TTAIC, the gene regulation in response to TTA pretreatment was mostly antagonistic to the effect found in $I C v s$. CTR (Figure 3).

Fatty acid metabolism and anti-inflammatory gene expression were less affected by pretreatment compared to treatment with TTA. The two fat metabolism related genes ANGPTL4 and elongation of very long chain fatty acids protein 2 (ELOV2 ) were both up-regulated. Further we observed increased transcription of Interleukin $17 \mathrm{D}($ IL 17D). The IL 17 family possesses an essential role in protecting the host from infection by pathogens [41]. The anti-inflammatory gene $P A C A$ and the pattern recognition receptor $N P T X 2$, both described in the previous contrasts showed increased gene expression. It should be noted that the gene regulation of NPTX2 in preTTA vs. CTR was opposite to that found in TTA vs CTR. Two transmembrane proteins, Integrin $\beta 5$ (ITB5) and thrombospondin-4 (thbs4b), both contributing to cell adhesion and migration showed increased transcription following pre-treatment with TTA. In addition we found increased expression of the transmembrane receptors roundabout-1 (ROBO1) and plexinC1 (PLXNC1). Both receptors are considered to be repulsive receptors, contributing to the release of cell adhesion upon ligand activation $[42,43]$. These results may indicate that pretreatement with TTA supports increased cell migration in ASK cells, similar to the effects found in TTA vs. CTR.

Approximately $10 \%$ of the genes DE following TTA pre-treatment were transporters/ion channels. The genes included the GABA transporter (SLC6A13), Na ${ }^{+}$/dicarboxylate co-transporter (SLC13A2), SLC22A15b,

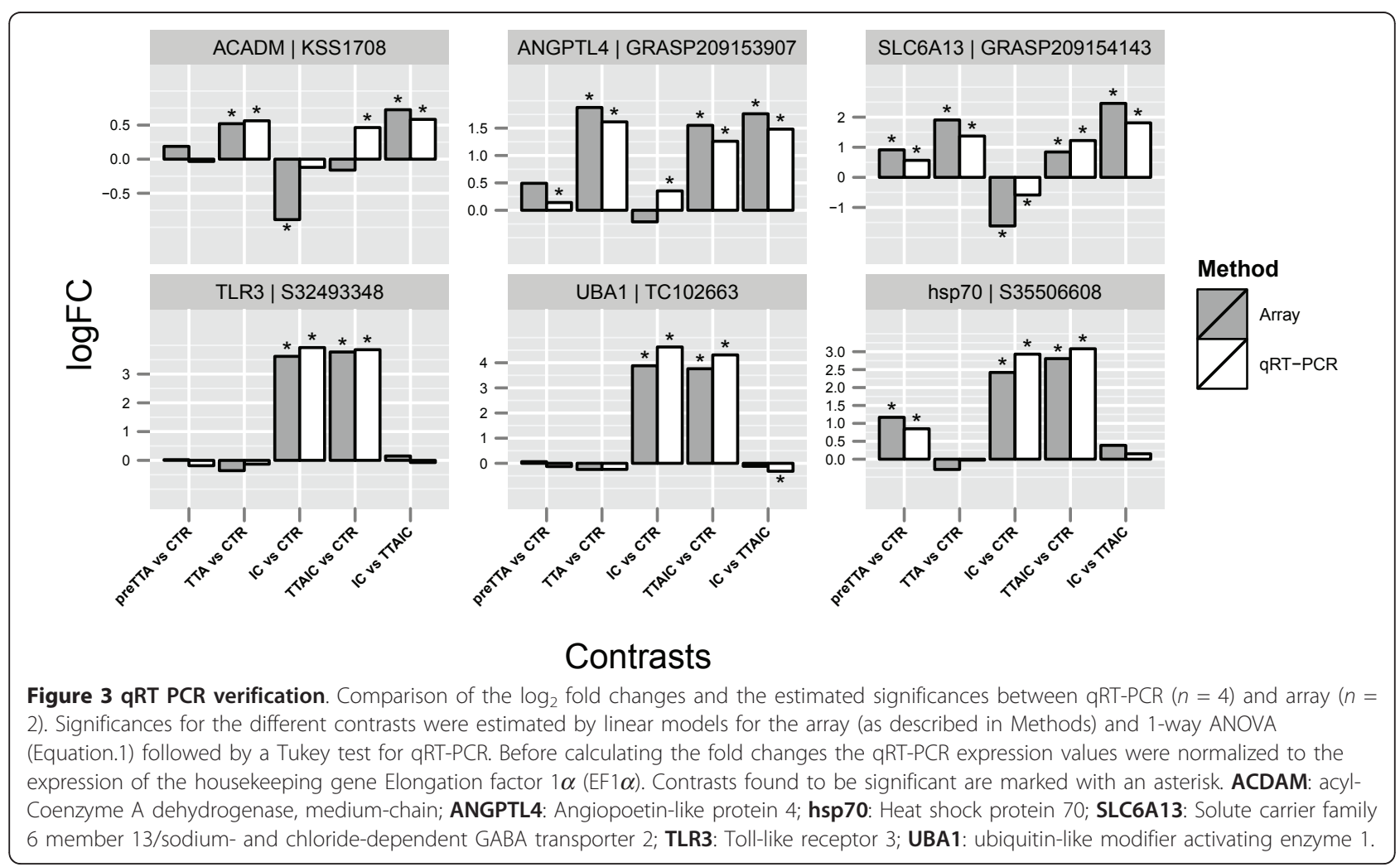


SLC43A3, $\mathrm{Cl}^{-}$transporter (CLCN5), v-type proton ATPase subunit s1 (ATP6AP1), ATP2A1, K-channel (KCNQ5), the neurotransmitter-gated ion channel GLRB and the $\mathrm{Ca}^{2+}$ activated chloride channel TTYH3 as the only one showing down-regulation. CLCN5 and ATP6AP1 are responsible for the acidification of the endosomal lumen. Hence, the increased expression suggests a higher capacity for acidification of the endosome, which may promote phagocytosis [44].

\section{qRT-PCR}

To validate the microarray results, six genes were randomly chosen from the list of DE genes for all contrasts. The genes were analyzed by qRT-PCR (Figure 4). In four cases we were able to detect significance by qRT-PCR, but not by array. However, in these cases the $\log _{2}$ FC proofed to be lower than the cut-off of 0.5 , that we used to identify significant genes in the array experiment. In one case we detected significance by array, but could not confirm this by qRT-PCR. Overall the results indicate that the parameters chosen to account for multiple hypothesis testing were appropriate. The results of the $\log _{2} \mathrm{FCs}$ for qRT-PCR matched those obtained by array. When comparing the $\log _{2} \mathrm{FCs}$ obtained for all genes and contrasts between qRT-PCR and array we found a highly significant correlation (pearson correlation of $\mathrm{r}=0.96, \mathrm{p}=2.2 \mathrm{e}^{-16}$ and an $\mathrm{R}^{2}=0.92$ ).

To further study immune modulatory effects of TTA in ASK cells, we used qRT-PCR to measure the expression of selected genes previously reported to respond directly to TTA or other PPAR agonists (Cox2, IL10, $P P A R \gamma, T N F \alpha, A r g 1)$ and/or are important mediators of inflammation (IL1 $\beta, I F \alpha(1+2), M x)$ (Figure 4). None of the analyzed genes was found to respond significantly to the effect of pretreatment with TTA. Several genes, however, responded significantly to the four different treatments.

For Interleukin 10 (IL10), which is generally considered to have anti-inflammatory properties, we found significant lower expression in the group that received poly (I:C), compared to all other treatment groups. The two pro-inflammatory cytokines TNF $\alpha$ and Interferon $\alpha$ showed no DE in response to the different treatments. It should be noted that the expression of TNF $\alpha$ could only be detected in poly(I:C) elicited samples and that concordant application of TTA and poly(I:C) reduced the mean expression by $50 \%$. PPAR $\gamma$ showed no altered expression in response to the different treatments. Arginase 1 was significantly higher expressed in all samples that received poly $(\mathrm{I}: \mathrm{C})$ treatment, additionally we observed that TTA also increased the Arginase 1

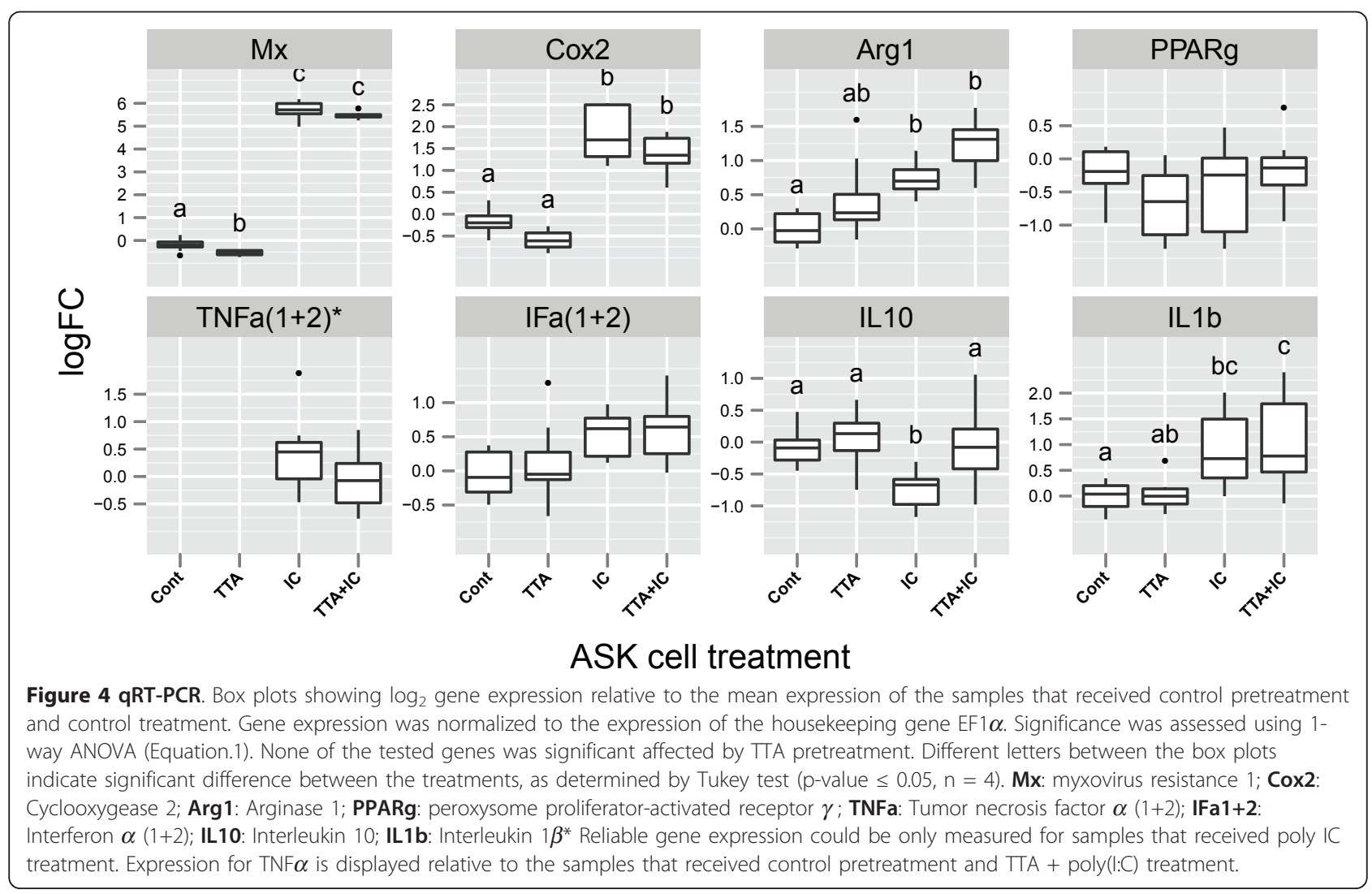


expression. The mean expression of the samples receiving TTA and poly(I:C) was $67 \%$ higher compared to the poly(I:C) samples. Even though not statistical significant, we observed a reduction in the expression of $C O X 2$ upon TTA treatment. Interleukin $1 \beta$ is considered to be pro-inflammatory. We found a significant increase in transcription upon poly(I:C) elicitation, while concordant treatment with TTA and poly(I:C) elevated the mean expression (not significant). $\mathrm{Mx}$ is considered to be an antiviral protein [45], TTA treatment caused a significant reduction of $M x$ gene expression. Treatment with poly(I:C) increased the expression strongly.

\section{Discussion}

In the present study we characterized the transcriptional responses to the PPAR activator TTA in the Atlantic salmon macrophage-like ASK cell line. Considering the strong effects documented for various PPAR agonists in different tissues like intestine or fat tissue (reviewed [46]) we found relatively few genes responding with altered transcription to TTA treatment. However, based on the results of the Microarray and qRT-PCR analysis TTA was found to stimulate the capacity for fat metabolism and have anti-inflammtory effects in the ASK cell line.

The innate immune response recognizes invading pathogens by pattern recognition receptors (PRR) through recognition of pathogen-associated molecular patterns. Toll-like receptors are a family of PRRs where each receptor displays specificity for it's lig-and [47]. Viral pathogens produce double stranded (ds) RNA during one part of their reproduction cycle. DsRNA is recognized by TLR3, triggering the production and release of pro-inflammatory cytokines [48]. The results from our microarray study showed that indeed a high number of genes were DE in poly (I:C) elicited macrophages. By comparing the group that received concordant treatment with TTA and poly(I:C) against the group that received poly(I:C) alone we identified a subset of genes showing antagonistic regulation. Several of these genes were sparsely characterized in the literature, especially in relation to immune function. However, the fact that we found those genes to be induced by poly(I: C) suggests a function in the Atlantic salmon immune response. Indicating that application of TTA to Atlantic salmon ASK cells has immune modulatory properties.

Expression of anti-inflammatory markers by macrophages is a hallmark for the alternative activated state. Anti-inflammatory effects of TTA have been mainly ascribed to increased expression of anti-inflammatory IL10. Indeed, we found a significant $98 \%$ increased transcription of IL10. Hence, indicating that TTA increases expression of IL1O in poly(I:C) activated macrophages, similar to the effects reported from human PBMCs stimulated with TTA $[49,50]$.
Further we identified the $\mathrm{PGE}_{2}$ inhibitor KLF11 [23] as up-regulated in response to TTA. $\mathrm{PGE}_{2}$ is a mediator of inflammation, produced from $\mathrm{PGH}_{2}$ which is mainly produced by COX2. We observed that in poly(I:C) elicited ASK cells, TTA caused a mean reduction of $24 \%$ in $C O X 2$ gene expression. Arguably, these results indicate that TTA attenuates the capacity for production of pro-inflammatory $\mathrm{PGE}_{2}$, as it has been shown in primary macrophages from TTA fed fish reared at $5^{\circ} \mathrm{C}$, but not for those kept at $12^{\circ} \mathrm{C}$ [12].

Arg1 competes with iNOS, an enzyme controlling the production of Nitric oxide for it's substrate. Arg1 is considered to be a key marker for alternative activated macrophages [51]. Recent studies in mice have shown that the expression of Arg1 in alternative activated macrophages is essential for suppressing inflammation [52]. Thus, our observation that TTA increased the expression of Arg1 by 67\%, supports anti-inflammatory effects of TTA, and is also in accordance with a described regulation of Arg1 expression by PPAR- $\gamma$ and $\beta / \delta$ [51]. Studies conducted on murine macrophages have shown that an up-regulation in fatty acid oxidation is crucial to direct them towards an alternative activated state [53]. In agreement with this we found that TTA induced the expression of genes that are related to lipid metabolism in ASK cells. Namely we identified increased expression of Angiopoetin-like protein 4 (ANGPTL4), carnitine palmitoyltransferase 1 (Cpt1a), acyl-CoA dehydrogenase (Acdam), enoyl-Coenzyme A hydratase $(H A D H)$ and the lipid droplet associated protein PLIN2. These effects of TTA are also consistent with reports of increased fatty acid oxidation upon TTA treatment $[6,8]$ and also with effects associated to PPAR activation [5]. Taken together, the results provided by this study indicate that TTA directs the macrophagelike ASK cells towards an anti-inflammational, alternative activated state. Studies conducted in mice have reported that macrophages in the alternative activated state, via macrophage-parenchymal cell crosstalk, affect fat catabolism in liver and adipose tissues [54,55]. This effect was especially found to be important in animals under high-fat diet. Thus, we may speculate that TTA directed macrophage activation in Atlantic salmon fed high fat diets could contribute to an improved metabolic and inflammatory state.

Many studies have shown that the immune response is necessary in order to evict an invading pathogen, however, an excessive inflammatory response can cause unintended tissue damage, finally causing mortality [56]. Studies challenging Atlantic salmon with the viral pathogen ISAV (infectious salmon anemia virus) have shown that survival is not related to a strong innate inflammatory response, but rather to an attenuated innate and a stronger cell-mediated immune response 
[40]. Hence, it appears possible that the reduced mortality as reported from TTA fed Atlantic salmons during natural disease outbreaks $[10,13]$ may partly result from anti-inflammatory effects of TTA.

TTA has been reported to act as an agonist for all PPAR subtypes in vitro $[3,4]$, and it is assumed that most physiological effects of TTA are mediated via activation of PPARs. In our experiment we found neither significant transcriptional regulation of $P P A R-\alpha, \beta / \delta$ (represented on the Array, data not shown) nor PPAR- $\gamma$, (Figure 4). However, the fact that a large proportion of the genes identified in our study have been reported to be regulated by PPARs and that the list of DE genes shows considerable overlap with the results from PPAR stimulated murine macrophages K.O for PPAR- $\gamma$ or $\beta / \delta$ [57] support our assumption that the ASK polarization towards an alternative activated state may be induced via activation of PPAR $-\gamma$ and $\beta / \delta$ by TTA.

\section{Conclusions}

The results presented in this study suggests that TTA directs the macrophage-like cells towards an alternative activated state which is indicated by a stimulated lipid metabolism and attenuated inflammatory response. Further the pattern of TTA regulated genes indicate that the stimulation occurs via PPAR activation. The effects confirm a function of TTA in Atlantic salmon macrophages similar to those documented in mammals. These findings supports that TTA modulates the immune system of Atlantic salmon.

\section{Methods \\ Cell Culture}

The Atlantic salmon kidney cell line (ASK: ATCC: CRL2747) [14] was cultured and propagated at $20^{\circ} \mathrm{C}$ according to standard cell culture procedures. Compared to mammalian cell lines, fish cell lines thrive slowly [58], so the ASK cells were studied for the respectively long period of 7 days. The cells were seeded out at day 1 in 6 well format (density $5 \times 10^{4}$ cells $/ \mathrm{ml}$ ). On day $2-4$ the cells were pretreated with either TTA or control medium. Day 5-7: cells were treated with either Control, TTA, TTA+poly(I:C) or poly(I:C) medium.

Growth medium was L-15 (Invitrogen, MD, USA) supplemented with $10 \%$ FBS (Invitrogen), $40 \mu \mathrm{M} \beta$-Mercaptoethanol and $50 \mu \mathrm{g} / \mathrm{ml}$ Gentamycin. TTA medium was prepared by dissolving TTA (ThiaMed-ica, Norway) in $0.1 \mathrm{M} \mathrm{NaOH}$ at $80^{\circ} \mathrm{C}$ to a concentration of $100 \mathrm{mM}$, this stock-solution was diluted to a final concentration of $100 \mu \mathrm{M}$ in growth-medium [59]. Control-medium was obtained by adding the same volume of $0.1 \mathrm{M} \mathrm{NaOH}$ as used in the TTA medium to the growth medium. Poly $\mathrm{I}: \mathrm{C}$ was prepared by adding poly(I:C) (invitrogen) to a final concentration of $(50 \mu \mathrm{g} / \mathrm{ml})$ to Control-medium.
TTA+poly(I:C) medium was obtained by adding poly(I: C) to a final concentration of $(50 \mu \mathrm{g} / \mathrm{ml})$ to the TTAmedium.

The cells were harvested on day 7 for RNA extraction. An overview of the experimental design is given in Table 1. The experiment was repeated four times with four different passages (passages 6-9) from the cells.

\section{RNA extraction}

Total RNA was extracted using Qiazol (Qiagen, Halle, Germany) and purified from the cells using column purification (Qiagen) according to the manufacturer's instructions. Traces of genomic DNA in the samples were eliminated by on-column-DNase (Qiagen) digestion. RNA concentrations were measured for all samples using a NanoDrop 1000 Spectrophotometer. RNA quality for samples later used in the Microarray was determined using a Agilent 2100 Bioanalyzer (RNA 6000 NanoLabChip, Agilent, CA, USA). All samples had RIN values ranging from 9.6 to 10 , indicating high quality of the RNA. Only individual RNA samples were used for subsequent qRT-PCR and microarray experiments.

\section{Quantitative RT-PCR}

Single strand CDNA was reverse transcribed from 500 ng of total, DNase treated RNA using oligo dT primers and the Taq Man reverse transcription Kit (Applied Biosystems, Tx, USA). qRT-PCR was performed in 96-well optical plates on a Light-Cycler 480 (Roche, Switzerland). For the PCR reaction $2 \times$ SYBR green I master Mix (Roche), $0.41 \mathrm{nM}$ of each primer and the cDNA template were mixed in a total reaction volume of $10 \mu \mathrm{l}$. Primer sequences are listed in Table 4. A three step PCR protocol with 45 cycles $\left(15 \mathrm{~s} 95^{\circ} \mathrm{C}, 15 \mathrm{~s} 60^{\circ} \mathrm{C}, 15 \mathrm{~s}\right.$ $72^{\circ} \mathrm{C}$ ) was used. To verify specific amplification a melting curve analysis step was done at the end of the program. All samples were analyzed in duplicates and for each measured gene a standard curve was produced using a serial dilution from a pool of all cDNA samples.

The expression levels were calculated using the standard curve method (Applied Biosystems User Bulletin $2)$. Expression levels were standardized to the expression of the housekeeping gene Elongation factor $1 \alpha(\mathrm{EF} 1 \alpha$, $[60]$ ), and $\log _{2}$ FCs were calculated relative to the average standardized sample that received control pretreatment and control treatment. Significance was tested by conducting a analysis of variance (ANOVA) test on the $\log _{2}$ FCs for each gene using the model:

$$
y_{i j k}=\mu+P_{j}+T_{k}+\varepsilon_{i j k}
$$

Where $y_{i j k}$ is the $\log _{2} \mathrm{FC}$ of the $i^{\text {th }}$ sample that received pretreatment $j$ and treatment $k . P_{j}$ is the effect of pretreatment $j=\{$ contr, TTA $\}, T_{k}$ is the effect of treatment 
Table 4 qRT-PCR primer sequences

\begin{tabular}{|c|c|c|c|}
\hline ID/Gene.Acc.Nr. & Gene & Forward primer & Reverse primer \\
\hline AF321836 & $\mathrm{EF} 1 \alpha$ & CACCACCGGCCATCTGATCTACAA & TCAGCAGCCTCCTTCTCGAACTTC \\
\hline KSS1708 & ACADM & GCCATCTCAGCCAACAGGAA & GACAAGACCAGACCGCCAGT \\
\hline GRASP209147493 & S6A13 & CCGTATGGGGGATGATGCTAA & GGTAGTATGCTGACGACTGACACCT \\
\hline S35506608 & HSP70 & AAGGCAAGATCAGCGAGGAG & TGCCTGATCTCCACAGCAAC \\
\hline GRASP209153907 & ANGL4 & AGGAGATGCAGCAGGAGAGG & TTCTGAGCTTCCAGCATCCA \\
\hline S32493348 & TLR3 & TCCAAGCCTGGTGACTCTCA & CAGGTGGAGGACTCGTTAGGG \\
\hline TC102663 & UBA1 & GCACTGTTTCCCCTCTGACC & AGCAGTCTCGTGTTCCCACA \\
\hline AF321836 & $\mathrm{TNF} \alpha(1+2)$ & GCTTGTCTCTTGTTGCCACCA & TGTGTGGGATGAGGATTTGGTT \\
\hline AF088999 & $\operatorname{cox} 2$ & ACCTATGGGAACCGCAGGA & GTGAAGTGCTGGGCGAAGA \\
\hline EG929369 & ARG1 & AGCCATGCGTATCAGCCAA & AAGGCGATCCACCTCAGTCA \\
\hline AJ416951 & PPAR $\gamma$ & CATTGTCAGCCTGTCCAGAC & TTGCAGCCCTCACAGACATG \\
\hline EF165028 & IL10 & ATGAGGCTAATGACGAGCTGGAGA & GGTGTAGAATGCCTTCGTCCAACA \\
\hline AY216594+AY216595 & $\mid F \alpha(1+2)$ & TGCAGTATGCAGAGCGTGTG & TCTCCTCCCATCTGGTCCAG \\
\hline SSU66477 & $\mathrm{MX}$ & TGATCGATAAAGTGACTGCATTCA & TGAGACGAACTCCGCTTTTCA \\
\hline AY617117 & $\operatorname{IL} 1 \beta$ & AGCAGGGTTCAGCAGTACAT & CTCCATAGCCTCACTCATCA \\
\hline
\end{tabular}

$k=\{$ contr, TTA, TTA+polyI:C, polyI:C $\}, \mu$ is a constant and $\varepsilon$ is the error. A Tukey test was used subsequently to identify differences within significant effects.

\section{Microarray hybridization}

The customized $4 \times 44 \mathrm{~K}$ oligo (60-mer) Atlantic salmon microarrays SIQ2.0 (Agilent) [18] was used in the experiment. Unless otherwise stated all equipment and reagents were from Agilent. Amplification and labeling of $500 \mathrm{ng}$ of total RNA and spike-ins for each sample was performed using the Quick Amp Labeling Kit (OneColor). After fragmentation of the Cy3 labeled cRNA, the samples were hybridized to the microarray at $65^{\circ} \mathrm{C}$ for $17 \mathrm{~h}$. After hybridization the arrays were washed with Gene Expression wash Buffer 1 and 2 and scanned using a Agilent Microarray Scanner. All steps were conducted according to the Agilent protocol (One-Color Quick Amp Labeling, Version 5.7). Analysis of the microarray images was done in Agilent Feature Extraction (Version 10.7.1.1) Software using the one-color (GE1_107_Sep09) protocoll. Array quality was assessed through Agilent control features and spike-in controls.

\section{Data analysis}

One array did not pass the quality checks and was excluded from subsequent analysis. Normalization and analysis of the data was done in R/Bioconductor [61] using the Bioconductor package "limma" [19]. To generate the data-set, background corrected spot intensity signals (gProcessedSignal) were filtered according to the following criteria provided by Feature Extraction: gIsFound, gIsPosAndSignif, gIsWellAboveBG and ControlType (a description of the feature results can be found in the Feature Extraction Software Reference Guide). All Control spots were removed from the data set. The mean of the intensity signal for the duplicated features was calculated and the data was subsequently normalized by quantile normalization in order to adjust the scale of intensities across arrays [62]. The data was then $\log _{2}$ transformed and probe sets showing more than two missing values were discarded from the data set, classifying 12496 (59\%) probe sets as present. Processed and raw microarray data is publicly available at NCBI's GEO repository (http://www.ncbi.nlm.nih.gov/geo/, Acc.Nr: GSE25328). A linear model (using zero intercept parameterization) was fitted to each probe set, where $y_{i j k l}$ is the $\log _{2}$ intensity of the $i^{t h}$ sample that received pretreatment $j$ and treatment $k . P_{j}$ is the effect of pretreatment $j=\{$ contr, TTA $\}, T_{k}$ is the effect of treatment $k=$ \{contr, TTA, TTA+polyI:C, polyI:C\}, $B_{l}$ is the batch effect $l=\{1,2\}, \mu$ is a constant and $\varepsilon$ is the error.

$$
y_{i j k l}=P_{j}+T_{k}+B_{l}+\varepsilon_{i j k l}
$$

Subsequent to fitting the model to each probe set, a number of specific contrasts of interest (listed in Table 2) were extracted for each probe set. For a graphical representation of the samples that were compared by the contrasts see Additional file 2, Figure S1. Improved variance estimates for the contrasts were computed using empirical Bayes moderated t-statistics from the "limma" package [63]. Before accounting for multiple hypothesis testing, probe sets without annotation were removed, reducing the data set to 10627 (50\%) probe sets. Multiple hypothesis testing was accounted for by controlling the false discovery rate (FDR) [64] across all probes and contrasts simultaneously (using the method: global in the decideTests function from the "limma" package). Probes were declared significant when the $q$-value was $\leq 0.15$ and the $\log _{2} \mathrm{FC} \geq 0.5$. 
Annotations for the probes were obtained using the function topBlast from the program Blast2GO [65]. Full length cDNA sequences of the probes have been compared in a BlastX search against the Swissprot protein database using an E-value cut-off of $10^{-6}$ and the default program settings. All plots were generated using the $\mathrm{R}$ package "ggplot2" [66] and heatmaps were produced using "lattice" [67].

\section{Additional material}

Additional file 1: Table S1. Table(.csv) of all probes. Column 1: Probe ID; columns 2-6: $\log _{2}$ FCs of the corresponding gene for the 5 different contrasts preTTA vs. CTR, TTA vs. CTR, TTAIC vs. CTR, IC vs. CTR and IC vs. TTAIC. Column 7-9: Gene annotation, gene symbol and e-value. Column 10-14: significance of the corresponding gene for the corresponding contrast.

Additional file 2: Figure S1. Contrasts: A: Figure of the experimental design (Table 1). Each of the eight nodes corresponds to a unique combination of the two experimental factors Pretraetment and

Treatment. B: Figure displaying which combinations of Pretreatment and Treatment were used in the contrast preTTA vs. CTR (RED nodes vs. BLUE nodes). Figure for the remaining four contrasts are displayed; $\mathbf{C}$ : TTA vs. CTR D: IC VS. CTR E: TTAIC vs. CTR F: IC vs. TTAIC. Comparison is RED nodes vs. BLUE nodes, GREY nodes are not considered for the corresponding contrast.

\section{Acknowledgements}

The work was funded by the Research Council of Norway (NRC project no: 179481/I30). We would like to thank Dr. Torben Luders from the Institutt for klinisk epidemiologi og molekylrbiologi (EpiGen) for his support in scanning the microarrays. Also we would like to thank Dr. Aleksei Krasnov (Nofima) for providing the microarray platform and Carlos Lozano (AFGC) for his help with the analysis.

\section{Author details}

${ }^{1}$ Institute of Animal and Aquaculture Sciences, Norwegian University of Life Sciences, P.O. Box 5003, N-1432 As-UMB, Norway. ${ }^{2}$ NOFIMA, P.O. Box 5010, N-1432 s, Norway. ${ }^{3}$ AVS Chile SA, Casilla 300, Puerto Varas, Chile.

\section{Authors' contributions}

FG designed and conducted the experiment, analysed the data and drafted the manuscript. HT participated in design of the study and writing the manuscript. Both authors read and approved the final manuscript.

Received: 7 December 2010 Accepted: 20 July 2011

Published: 20 July 2011

\section{References}

1. Sargent JR, Tocher DR, Bell JG: The Lipids. In Fish Nutrition.. 3 edition. Edited by: Halver JE, Hardy RW. London: Academic Press; 2002:181-257.

2. Herme G, Sandnes K: Effect of dietary lipid level on muscle composition in Atlantic salmon Salmo salar. Aquaculture Nutrition 1999, 5:9-16.

3. Berge K, Tronstad KJ, Flindt EN, Rasmussen TH, Madsen L, Kristiansen K, Berge RK: Tetradecylthioacetic acid inhibits growth of rat glioma cells ex vivo and in vivo via PPAR-dependent and PPAR-independent pathways. Carcinogenesis 2001, 22(11):1747-55.

4. Westergaard $M$, Henningsen J, Svendsen $M L$, Johansen $C$, Jensen UB, Schrøder HD, Kratchmarova I, Berge RK, Iversen L, Bolund L, Kragballe K, Kristiansen K: Modulation of keratinocyte gene expression and differentiation by PPAR-selective ligands and tetradecylthioacetic acid. J Invest Dermatol 2001, 116(5):702-12.
5. Chinetti G, Fruchart JC, Staels B: Peroxisome proliferator-activated receptors (PPARs): nuclear receptors at the crossroads between lipid metabolism and inflammation. Inflamm Res 2000, 49(10):497-505.

6. Berge RK, Skorve J, Tronstad KJ, Berge K, Gudbrandsen OA, Grav H: Metabolic effects of thia fatty acids. Curr Opin Lipidol 2002, 13(3):295-304

7. Skrede S, Sørensen HN, Larsen LN, Steineger HH, Høvik K, Spydevold OS, Horn R, Bremer J: Thia fatty acids, metabolism and metabolic effects. Biochim Biophys Acta 1997, 1344(2):115-31.

8. Kennedy S, Bickerdike R, Berge R, Dick J, Tocher D: Influence of conjugated linoleic acid (CLA) or tetradecylthioacetic acid (TTA) on growth, lipid composition, fatty acid metabolism and lipid gene expression of rainbow trout (Oncorhynchus mykiss L.). Aquaculture 2007, 272(14):489-501.

9. Kleveland EJ, Ruyter B, Vegusdal A, Sundvold H, Berge RK, Gjøen T: Effects of 3-thia fatty acids on expression of some lipid related genes in Atlantic salmon (Salmo salar L.). Comp Biochem Physiol B, Biochem Mol Biol 2006, 145(2):239-48.

10. Rørvik KA, Alne H, Gaarder M, Ruyter B, Måseide NP, Jakobsen JV, Berge RK, Sigholt T, Thomassen MS: Does the capacity for energy utilization affect the survival of post-smolt Atlantic salmon, Salmo salar L., during natural outbreaks of infectious pancreatic necrosis? Journal of Fish Diseases 2007, 30(7):399-409.

11. Moya-Falcón C, Hvattum E, Dyrøy E, Skorve J, Stefansson SO, Thomassen MS, Jakobsen JV, Berge RK, Ruyter B: Effects of 3-thia fatty acids on feed intake, growth, tissue fatty acid composition, betaoxidation and $\mathrm{Na}+, \mathrm{K}+-\mathrm{ATPase}$ activity in Atlantic salmon. Comp Biochem Physiol B, Biochem Mol Biol 2004, 139(4):657-68.

12. Gjøen T, Kleveland EJ, Moya-Falcón C, Frøystad MK, Vegusdal A, Hvattum E, Berge RK, Ruyter B: Effects of dietary thia fatty acids on lipid composition, morphology and macrophage function of Atlantic salmon (Salmo salar L.) kidney. Comp Biochem Physiol B, Biochem Mol Biol 2007 148:103-11.

13. Alne H, Thomassen MS, Takle H, Terjesen BF, Grammes F, Oehme M, Refstie S, Sigholt T, Berge RK, Rørvik KA: Increased survival by feeding tetradecylthioacetic acid during a natural outbreak of heart and skeletal muscle inflammation in So Atlantic salmon, Salmo salar L. Journal of Fish Diseases 2009, 32(11):953-61.

14. Devold M, Krossøy B, Aspehaug V, Nylund A: Use of RT-PCR for diagnosis of infectious salmon anaemia virus (ISAV) in carrier sea trout Salmo trutta after experimental infection. Dis Aquat Org 2000, 40:9-18.

15. Ellis $A E$ : Innate host defense mechanisms of fish against viruses and bacteria. Dev Comp Immunol 2001, 25(8-9):827-39.

16. Gordon S: Alternative activation of macrophages. Nat Rev Immunol 2003, 3:23-35.

17. Weber F, Wagner V, Rasmussen SB, Hartmann R, Paludan SR: Doublestranded RNA is produced by positive-strand RNA viruses and DNA viruses but not in detectable amounts by negative-strand RNA viruses. J Virol 2006, 80(10):5059-64.

18. Krasnov A, Timmerhaus G, Afanasyev S, Jørgensen SM: Development and assessment of oligonucleotide microarrays for Atlantic salmon (Salmo salar L.). Comparative biochemistry and physiology Part D, Genomics \& proteomics 2010

19. Smyth GK: Limma: linear models for microarray data. In Bioinformatics and Computational Biology Solutions using $R$ and Bioconductor. Edited by: Gentleman R, Carey V, Dudoit S, R Irizarry WH. New York: Springer; 2005:397-420.

20. Mandard S, Zandbergen F, van Straten E, Wahli W, Kuipers F, Müller M, Kersten S: The fasting-induced adipose factor/angiopoietin-like protein 4 is physically associated with lipoproteins and governs plasma lipid levels and adiposity. The Journal of Biological Chemistry 2006, 281(2):934-44.

21. Lu B, Jiang YJ, Zhou Y, Xu FY, Hatch GM, Choy PC: Cloning and characterization of murine 1-acyl-sn-glycerol 3-phosphate acyltransferases and their regulation by PPARalpha in murine heart. Biochem J 2005, 385(Pt 2):469-77.

22. Persson J, Degerman E, Nilsson J, Lindholm MW: Perilipin and adipophilin expression in lipid loaded macrophages. Biochemical and Biophysical Research Communications 2007, 363(4):1020-6.

23. Buttar NS, DeMars CJ, Lomberk G, Rizvi S, Bonilla-Velez J, Achra S, Rashtak S, Wang KK, Fernandez-Zapico ME, Urrutia R: Distinct role of Kruppellike factor 11 in the regulation of prostaglandin E2 biosynthesis. The Journal of Biological Chemistry 2010, 285(15):11433-44 
24. Bivol LM, Hultström M, Gudbrandsen OA, Berge RK, Iversen BM: Tetradecylthioacetic acid downregulates cyclooxygenase 2 in the renal cortex of two-kidney, one-clip hypertensive rats. Am J Physiol Regul Integr Comp Physiol 2008, 295(6):R1866-73.

25. Fazio S, Babaev VR, Murray AB, Hasty AH, Carter KJ, Gleaves LA, Atkinson JB, Linton MF: Increased atherosclerosis in mice reconstituted with apolipoprotein E null macrophages. Proc Natl Acad Sci USA 1997, 94(9):4647-52.

26. liang Zhang $\mathrm{H}, \mathrm{Wu} J$, Zhu J: The role of apolipoprotein E in Guillain-Barré syndrome and experimental autoimmune neuritis. J Biomed Biotechnol 2010, 2010:357412.

27. Akiyama TE, Sakai S, Lambert G, Nicol CJ, Matsusue K, Pimprale S, Lee YH, Ricote M, Glass CK, Brewer HB, Gonzalez FJ: Conditional disruption of the peroxisome proliferator-activated receptor gamma gene in mice results in lowered expression of $A B C A 1, A B C G 1$, and apoE in macrophages and reduced cholesterol efflux. Molecular and Cellular Biology 2002, 22(8):2607-19

28. Gourdine JP, Markiv A, Smith-Ravin J: The three-dimensional structure of codakine and related marine C-type lectins. Fish \& Shellfish Immunology 2007, 23(4):831-9.

29. Martin SAM, Blaney SC, Houlihan DF, Secombes CJ: Transcriptome response following administration of a live bacterial vaccine in Atlantic salmon (Salmo salar). Mol Immunol 2006, 43(11):1900-11.

30. O'Farrell C, Vaghefi N, Cantonnet M, Buteau B, Boudinot P, Benmansour A: Survey of transcript expression in rainbow trout leukocytes reveals a major contribution of interferon-responsive genes in the early response to a rhabdovirus infection. J Virol 2002, 76(16):8040-9.

31. Magnadóttir B: Innate immunity of fish (overview). Fish \& Shellfish Immunology 2006, 20(2):137-51

32. Delgado M, Abad C, Martinez C, Juarranz MG, Leceta J, Ganea D, Gomariz RP: PACAP in immunity and inflammation. Ann N Y Acad Sci 2003, 992:141-57.

33. Simonet WS, Lacey DL, Dunstan CR, Kelley M, Chang MS, Lüthy R, Nguyen $\mathrm{HQ}$, Wooden $\mathrm{S}$, Bennett L, Boone T, Shimamoto G, DeRose M, Elliott R, Colombero A, Tan HL, Trail G, Sullivan J, Davy E, Bucay N, Renshaw-Gegg L, Hughes TM, Hill D, Pattison W, Campbell P, Sander S, Van G, Tarpley J, Derby P, Lee R, Boyle WJ: Osteoprotegerin: a novel secreted protein involved in the regulation of bone density. Cell 1997, 89(2):309-19

34. Zauli G, Corallini F, Bossi F, Fischetti F, Durigutto P, Celeghini C, Tedesco F, Secchiero P: Osteoprotegerin increases leukocyte adhesion to endothelial cells both in vitro and in vivo. Blood 2007, 110(2):536-43.

35. Yin F, Banerjee R, Thomas B, Zhou P, Qian L, Jia T, Ma X, Ma Y, ladecola C, Beal MF, Nathan C, Ding A: Exaggerated inflammation, impaired host defense, and neuropathology in progranulin-deficient mice. J Exp Med 2010, 207:117-28.

36. Elkington PTG, O'Kane CM, Friedland JS: The paradox of matrix metalloproteinases in infectious disease. Clin Exp Immunol 2005, 142:12-20.

37. Luster $A D$, Rothenberg ME: Role of the monocyte chemoattractant protein and eotaxin subfamily of chemokines in allergic inflammation. $J$ Leukoc Biol 1997, 62(5):620-33.

38. Lee BC, Avraham S, Imamoto A, Avraham HK: Identification of the nonreceptor tyrosine kinase MATK/CHK as an essential regulator of immune cells using Matk/CHK-deficient mice. Blood 2006, 108(3):904-7.

39. Schiøtz BL, Jørgensen SM, Rexroad C, Giøen T, Krasnov A: Transcriptomic analysis of responses to infectious salmon anemia virus infection in macrophage-like cells. Virus Res 2008, 136(1-2):65-74

40. Jørgensen SM, Afanasyev S, Krasnov A: Gene expression analyses in Atlantic salmon challenged with infectious salmon anemia virus reveal differences between individuals with early, intermediate and late mortality. BMC Genomics 2008, 9:179

41. Onishi RM, Gaffen SL: Interleukin-17 and its target genes: mechanisms of interleukin-17 function in disease. Immunology 2010, 129(3):311-21.

42. Prasad A, Qamri Z, Wu J, Ganju RK: Slit-2/Robo-1 modulates the CXCL12/ CXCR4-induced chemotaxis of T cells. J Leukoc Biol 2007, 82(3):465-76.

43. Walzer T, Galibert L, Smedt TD: Dendritic cell function in mice lacking Plexin C1. Int Immunol 2005, 17(7):943-50.

44. Christensen El, Devuyst O, Dom G, Nielsen R, der Smissen PV, Verroust P, Leruth M, Guggino WB, Courtoy PJ: Loss of chloride channel CIC-5 impairs endocytosis by defective trafficking of megalin and cubilin in kidney proximal tubules. Proc Natl Acad Sci USA 2003, 100(14):8472-7.

45. Haller O, Stertz S, Kochs G: The Mx GTPase family of interferon-induced antiviral proteins. Microbes Infect 2007, 9(14-15):1636-43.

46. Bunger M, Hooiveld G, Kersten S, Muller M: Exploration of PPAR functions by microarray technology-A paradigm for nutrigenomics. Biochimica et Biophysica Acta (BBA) - Molecular and Cell Biology of Lipids 2007, 1771(8):1046-1064

47. Akira S, Uematsu S, Takeuchi O: Pathogen recognition and innate immunity. Cell 2006, 124(4):783-801.

48. Alexopoulou L, Holt AC, Medzhitov R, Flavell RA: Recognition of doublestranded RNA and activation of NF-kappaB by Toll-like receptor 3. Nature 2001, 413(6857):732-8.

49. Pettersen RJ, Muna ZA, Kuiper KK, Svendsen E, Müller F, Aukrust P, Berge RK Nordrehaug JE: Sustained retention of tetradecylthioacetic acid after local delivery reduces angioplasty-induced coronary stenosis in the minipig. Cardiovascular Research 2001, 52(2):306-13.

50. Aukrust P, Wergedahl H, Müller F, Ueland T, Dyrøy E, Damås JK, Frøland SS, Berge RK: Immunomodulating effects of 3-thia fatty acids in activated peripheral blood mononuclear cells. Eur J Clin Invest 2003, 33(5):426-33.

51. Odegaard JI, Ricardo-Gonzalez RR, Goforth MH, Morel CR, Subramanian V, Mukundan L, Eagle AR, Vats D, Brombacher F, Ferrante AW, Chawla A: Macrophage-specific PPARgamma controls alternative activation and improves insulin resistance. Nature 2007, 447(7148):1116-20.

52. Pesce JT, Ramalingam TR, Mentink-Kane MM, Wilson MS, Kasmi KCE, Smith AM, Thompson RW, Cheever AW, Murray PJ, Wynn TA: Arginase-1expressing macrophages suppress Th2 cytokine-driven inflammation and fibrosis. PLOS Pathog 2009, 5(4):e1000371.

53. Vats D, Mukundan L, Odegaard Jl, Zhang L, Smith KL, Morel CR, Wagner RA, Greaves DR, Murray PJ, Chawla A: Oxidative metabolism and PGC-1 beta attenuate macrophage-mediated inflammation. Cell Metabolism 2006, 4:13-24.

54. Odegaard JI, Ricardo-Gonzalez RR, Eagle AR, Vats D, Morel CR, Goforth $M H$, Subramanian V, Mukundan L, Ferrante AW, Chawla A: Alternative M2 activation of Kupffer cells by PPARdelta ameliorates obesity-induced insulin resistance. Cell Metabolism 2008, 7(6):496-507.

55. Kang K, Reilly SM, Karabacak V, Gangl MR, Fitzgerald K, Hatano B, Lee CH: Adipocyte-derived Th2 cytokines and myeloid PPARdelta regulate macrophage polarization and insulin sensitivity. Cell Metabolism 2008, 7(6):485-95.

56. Medzhitov R: Inflammation 2010: new adventures of an old flame. Cell 2010, 140(6):771-6.

57. Welch JS, Ricote M, Akiyama TE, Gonzalez FJ, Glass CK: PPARgamma and PPARdelta negatively regulate specidic subsets of lipopolysaccharide and IFN-gamma target genes in macrophages. Proc Natl Acad Sci USA 2003, 100(11):6712-7.

58. Fryer JL, Lannan CN: Three decades of fish cell culture: A current listing of cell lines derived from fishes. Journal of Tissue Culture Methods 1994, 16:87-94.

59. Tronstad KJ, Berge K, Flindt EN, Kristiansen K, Berge RK: Optimization of methods and treatment conditions for studying effects of fatty acids on cell growth. Lipids 2001, 36(3):305-13.

60. Olsvik PA, Lie KK, Jordal AEO, Nilsen TO, Hordvik I: Evaluation of potential reference genes in real-time RT-PCR studies of Atlantic salmon. BMC Mol Biol 2005, 6:21

61. Gentleman RC, Carey VJ, Bates DM, et al: Bioconductor: Open software development for computational biology and bioinformatics. Genome Biology 2004, 5:R80

62. Bolstad BM, Irizarry RA, Astrand M, Speed TP: A comparison of normalization methods for high density oligonucleotide array data based on variance and bias. Bioinformatics 2003, 19(2):185-93.

63. Smyth GK: Linear models and empirical bayes methods for assessing differential expression in microarray experiments. Stat Appl Genet Mol Biol 2004, 3:Article3.

64. Benjamini $\mathrm{Y}$, Yosef $\mathrm{H}$ : Controlling the false discovery rate: a practical and powerful approach to multiple testing. Journal of the Royal Statistical Society, Series B (Methodological) 1995, 57:289-300.

65. Conesa A, Götz S, García-Gómez JM, Terol J, Talón M, Robles M: Blast2GO: a universal tool for annotation, visualization and analysis in functional genomics research. Bioinformatics 2005, 21(18):3674-6. 
66. Wickham H: Ggplot: Elegant graphics for data analysis New York: Springer; 2009

67. Sarkar D: Lattice: Multivariate Data Visualization with R New York: Springer; 2008.

doi:10.1186/1471-2172-12-41

Cite this article as: Grammes and Takle: Anti-inflammatory effects of tetradecylthioacetic acid (TTA) in macrophage-like cells from Atlantic salmon (Salmo salar L.). BMC Immunology 2011 12:41.

Submit your next manuscript to BioMed Central and take full advantage of:

- Convenient online submission

- Thorough peer review

- No space constraints or color figure charges

- Immediate publication on acceptance

- Inclusion in PubMed, CAS, Scopus and Google Scholar

- Research which is freely available for redistribution

Submit your manuscript at www.biomedcentral.com/submit 